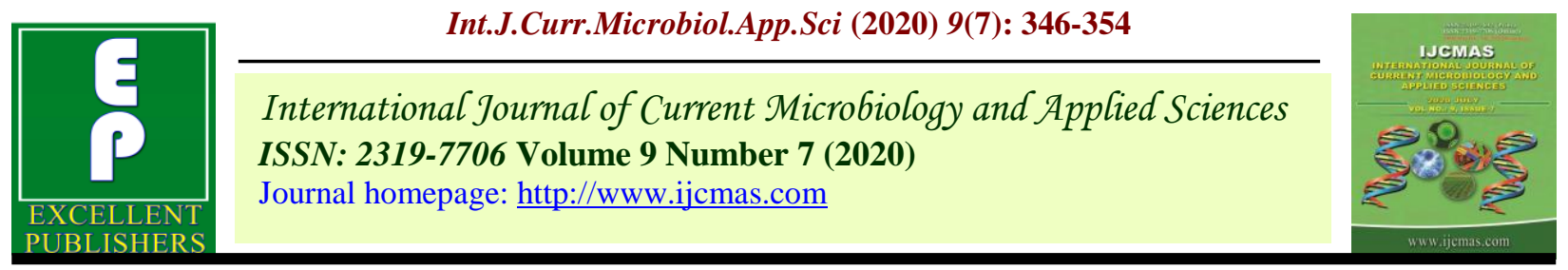

Original Research Article

https://doi.org/10.20546/ijcmas.2020.907.037

\title{
In-Vitro Efficacy of Forms and Methods of Adhatoda vasica against Flacherie causing Bacteria of Silkworm, Bombyx mori L.
}

\author{
K. V. Anitharani*, R. N. Bhaskar and H. G. Anusha \\ Department of Sericulture, UAS GKVK, Bengaluru-560065 (Karnataka), India \\ *Corresponding author
}

\begin{tabular}{|l|}
\hline Ke y w or d s \\
$\begin{array}{l}\text { Adhatodavasica, } \\
\text { bacterial flacherie, } \\
\text { inhibition zone. }\end{array}$ \\
\hline Article Info \\
\hline $\begin{array}{l}\text { Accepted: } \\
\text { 05 June } 2020 \\
\text { Available Online: } \\
\text { 10 July } 2020\end{array}$ \\
\hline
\end{tabular}

\section{A B S T R A C T}

\section{Introduction}

Mulberry silkworm is being reared indoor, since they are highly susceptible to disease causing pathogens which lead to drastic reduction in cocoon production. Flacherieis a condition wherein the silkworms are infected by several species of bacteria viz., Streptococcus faecalis, Staphylococcus aureus, Serratia sp. and Bacillus sp. (Sugun, 2000). Recently, many attempts have been made on the use of plant extracts to combat microbial infections as reported in ancient ayurvedic compendium "Charaka Samhita" and Sushrita Samhita" (Chopra, 1982; Chatterjee and Pakrashi, 1991). Action of plant on microorganisms pathogenic to other animals and insects has been reported (Ray and Majumdar, 1974).

This has commercially been exploited and utilized for management of pest and diseases of silkworm. Hence, an experiment was drawn in this line as to assess the efficacy of 
application of Adhatoda vasica against bacterial pathogens causing flacherie in the mulberry silkworm, Bombyx mori L. The botanical extracted in aqueous, acetone and alcohol media were studied in-vitro.

\section{Materials and Methods}

Silkworms affected by flacherie disease were spotted, $1 \mathrm{ml}$ of haemolymph was collected and mixed with $9 \mathrm{ml}$ distilled water to make the stock suspension from which serial dilutions $\left(10^{-5}\right.$ and $\left.10^{-7}\right)$ were prepared using 9 $\mathrm{ml}$ sterile water blanks (Siromani et al., 1994; Nataraju et al., 1999).

From the serial dilution prepared, $0.5 \mathrm{ml}$ each was transferred to separate petridishes containing nutrient agar medium. The culture plates were incubated at $37^{\circ} \mathrm{C}$ and after 48 hours, the incubated bacterial culture was observed under microscope to know the type and nature of colony formed. Based on the shape, texture and colour, the colonies were selected and purified on nutrient agar plate. The purified bacterial isolates were identified as Bacillus sp., Staphylococcus sp.and Streptococcus sp. The pathogenicity of the individual bacterium was confirmed by the principle of Koch's postulates. These organisms were used for in-vitro studies (Figure-1).

The botanical screened against Bacillus sp., Streptococcus sp. and Staphylococcus sp., bacteria isolated from silkworm haemolymph was leaf extract of A.vasica (common name: Adusoge, Family: Acanthaceae).

\section{Preparation of herbal extracts}

\section{Herbal powder}

The extract of A.vasica (Adusoge) was prepared as per procedure adopted by Krishnaprasad et al., (1979). The leaves of
A.vasica were collected from 'Sanjeevinivatika' (Herbal Garden) Division of Horticulture, University of Agricultural Sciences, Bengaluru. The required quantity of fresh leaves of A.vasica were harvested and surface sterilized with $70 \%$ ethyl alcohol then washed with sterile distilled water and shade dried. The shade dried leaves were then powdered in electric blender at slow speed, sieved and kept stored in desiccators.

\section{Aqueous plant extract}

The required quantity of fresh leaves were harvested and surface sterilized with $70 \%$ ethyl alcohol then washed with sterile distilled water. Later, they were taken in pestle and mortar separately and $10 \mathrm{ml}$ of sterile distilled water was added to $1 \mathrm{~g}$ of leaf for maceration. The extract was squeezed through double layered muslin cloth and used as stock solution. Further, the same was diluted by using sterile distilled water to achieve different concentrations (Karthikairaj et al., 2013).

\section{Acetone herbal extract}

Ten grams of herbal powder of A.vesica was kept in a conical flask soaked with acetone for 6 hours under air tight condition. The content was then stirred for an hour in magnetic stirrer and filtered through a filter paper. The residual extract was collected in a flask and the solvent is allowed to evaporate at room temperature. The extract was then stored at 4 ${ }^{\circ} \mathrm{C}$ until use. The resultant residue was made up to required volume using double distilled water and used for the study (Karthikairaj et al., 2013).

\section{Alcoholic herbal extract}

Ten grams of botanical powder was packed separately in a burette column keeping glass wool at the bottom and filter paper rings on 
top. Alcoholic extract was collected from the leaf powder by pouring double distilled water and ethyl alcohol on top of the burette column. The collected extract was then concentrated in hot water bath and made up to $10 \mathrm{ml}$ with distilled water and stored in the deep freezer. Required quantities of this extract was taken and diluted with distilled water to get the required concentrations (Karthikairaj et al., 2013).

\section{Placement of paper discs}

Sterilized whatman No.1 filter paper discs of $5 \mathrm{~mm}$ diameter dipped in botanical extracts for 1 minute and drained by the edges of petriplate were placed at the centre of the petriplate. Four replications were maintained for each treatment, further control (distilled water+bacteria and distilled water) was used for comparison. The bacteria inoculated plates were incubated for 48 hours at room temperature. The diameter of the inhibition zone by different forms of botanicals against each bacterium was measured $(\mathrm{mm})$ and recorded on 24 and 48 hours of incubation.

$\mathrm{T}_{1}-$ Aqueous extract

$\mathrm{T}_{2}-$ Acetone extract

$\mathrm{T}_{3}-$ Alcoholic extract

$\mathrm{T}_{4}-$ Water

\section{Results and Discussion}

Zone of inhibition ( $\mathrm{mm}$ ) observed in different concentrations $(2 \%, 4 \%$ and 6\%) of Adhatoda vasica against Bacillus sp

The aqueous, acetone and alcoholic extracts of A.vasica at different concentrations (2, 4 and $6 \%$ ) revealed significant results on zone of inhibition of Bacillus sp. with the dilutions of $10^{-5}$ and $10^{-7}$. The maximum zone of inhibition was recorded against $\mathrm{T}_{3}=$ Alcohol +Spraying $(8.00 \mathrm{~mm}$ and $9.12 \mathrm{~mm})$ after 24 hours of incubation with bacterial dilution of
$10^{-5}$ and $10^{-7}$ Further, after 48 hours of incubation, Bacillus sp. of both the dilutions $\left(10^{-5}\right.$ and $\left.10^{-7}\right)$ exhibited the same trend of zone of inhibition viz., maximum in $\mathrm{T}_{3}(8.99$ and $9.62 \mathrm{~mm})$ and minimum of 7.66 and 8.46 mm., 8.25 and $8.94 \mathrm{~mm}\left(10^{-5}\right.$ and $\left.10^{-7}\right) \mathrm{inT}_{1}$ on 24 and 48 hours respectively (Table 1 and Figure 2). These results follow the same trend as that of (Karthikairaj et al., 2013) who reported maximum inhibition zone of (9.33, 11.27 and $12.13 \mathrm{~mm}$ ) microbes involved in flacherie disease of silkworm for alcoholic plant extracts whereas comparatively less for their aqueous counterparts (8.83, 9.77 and $10.73 \mathrm{~mm}$ ) at different concentrations of 50 , 100 and $150 \mu \mathrm{l}$ of Andrographis paniculata followed by Momardica charentia (7.83, $8.33,9.43$ and $6.77,8.13$ and $8.93 \mathrm{~mm}$ ) and Ocimum sanctum $(7.33,7.67,8.83$ and 7.17 , 7.33 and $8.30 \mathrm{~mm})$.

Zone of inhibition ( $\mathrm{mm})$ observed in different concentrations $(2 \%, 4 \%$ and 6 $\%$ ) of Adhatoda vasica against Staphylococcus sp.

The data pertaining to zone of inhibition at different concentrations of A.vasica against Staphylococcus sp., was found significant among forms, methods and concentration of botanical. However, as the concentration of botanicals increased (2, 4 and $6 \%$ ) at 24 hours of incubation in artificial media, it revealed increased zone of inhibition for inoculation dose of $10^{-7}(5.35,5.66$ and 6.07 $\mathrm{mm})$. The trend was same even at $10^{-5}$ dilution (4.97, 5.38 and $5.72 \mathrm{~mm}$ ) recorded for 2, 4 and 6 per cent botanical formulation and found significant. The forms of botanicals used after 24 and 48 hours for $10^{-5}$ and $10^{-7}$ inoculation doses revealed significant results, however maximum of 7.42 and 7.88., 7.84 and $8.63 \mathrm{~mm}$ zone of inhibition was recorded for $\mathrm{T}_{3}$ which was found comparatively more effective in inhibiting the growth of Staphylococcus. 
Table.1 Zone of inhibition (mm) observed in different concentrations (2\%, $4 \%$ and 6\%) of Adhatoda vasica against Bacillus

\begin{tabular}{|c|c|c|c|c|c|c|c|c|c|c|c|c|c|c|c|c|}
\hline \multirow{4}{*}{\begin{tabular}{|l} 
Dilution \\
$\begin{array}{l}\text { Conc. of } \\
\text { botanical }\end{array}$ \\
$\begin{array}{l}\text { Forms of } \\
\text { botanical }\end{array}$
\end{tabular}} & \multicolumn{8}{|c|}{24 hours } & \multicolumn{8}{|c|}{48 hours } \\
\hline & \multicolumn{4}{|c|}{$10^{-5}$} & \multicolumn{4}{|c|}{$10^{-7}$} & \multicolumn{4}{|c|}{$10^{-5}$} & \multicolumn{4}{|c|}{$10^{-7}$} \\
\hline & \multirow[t]{2}{*}{$2 \%$} & \multirow[t]{2}{*}{$4 \%$} & \multirow[t]{2}{*}{$6 \%$} & \multirow[t]{2}{*}{ Mean } & \multirow[t]{2}{*}{$2 \%$} & \multirow[t]{2}{*}{$4 \%$} & \multirow[t]{2}{*}{$6 \%$} & \multirow[t]{2}{*}{ Mean } & \multirow[t]{2}{*}{$2 \%$} & \multirow[t]{2}{*}{$4 \%$} & \multirow[t]{2}{*}{$6 \%$} & \multirow[t]{2}{*}{ Mean } & \multirow[t]{2}{*}{$2 \%$} & \multirow[t]{2}{*}{$4 \%$} & \multirow[t]{2}{*}{$6 \%$} & \multirow[t]{2}{*}{ Mean } \\
\hline & & & & & & & & & & & & & & & & \\
\hline $\mathrm{T}_{1}$ & $\begin{array}{c}6.87 \\
(2.71)\end{array}$ & $\begin{array}{c}7.37 \\
(2.80)\end{array}$ & $\begin{array}{c}8.75 \\
(3.04)\end{array}$ & $\begin{array}{c}7.66 \\
(2.85)\end{array}$ & $\begin{array}{c}7.50 \\
(2.82)\end{array}$ & $\begin{array}{c}8.37 \\
(2.97)\end{array}$ & $\begin{array}{c}9.50 \\
(3.16)\end{array}$ & $\begin{array}{c}8.46 \\
(2.99)\end{array}$ & $\begin{array}{c}7.37 \\
(2.80)\end{array}$ & $\begin{array}{c}8.12 \\
(2.93)\end{array}$ & $\begin{array}{c}9.25 \\
(3.12)\end{array}$ & $\begin{array}{c}8.25 \\
(2.95)\end{array}$ & $\begin{array}{c}8.00 \\
(2.91)\end{array}$ & $\begin{array}{c}8.87 \\
(3.06)\end{array}$ & $\begin{array}{c}9.95 \\
(3.23)\end{array}$ & $\begin{array}{c}8.94 \\
(3.07)\end{array}$ \\
\hline $\mathbf{T}_{2}$ & $\begin{array}{c}7.75 \\
(2.87)\end{array}$ & $\begin{array}{c}7.87 \\
(2.89)\end{array}$ & $\begin{array}{c}7.87 \\
(2.89)\end{array}$ & $\begin{array}{c}7.83 \\
(2.88)\end{array}$ & $\begin{array}{c}7.75 \\
(2.87)\end{array}$ & $\begin{array}{c}8.62 \\
(3.02)\end{array}$ & $\begin{array}{c}9.12 \\
(3.10)\end{array}$ & $\begin{array}{c}8.50 \\
(3.00)\end{array}$ & $\begin{array}{c}8.25 \\
(2.95)\end{array}$ & $\begin{array}{c}8.67 \\
(3.02)\end{array}$ & $\begin{array}{l}10.00 \\
(3.24)\end{array}$ & $\begin{array}{c}8.97 \\
(3.07)\end{array}$ & $\begin{array}{c}8.50 \\
(3.00)\end{array}$ & $\begin{array}{c}9.50 \\
(3.16)\end{array}$ & $\begin{array}{l}10.50 \\
(3.31)\end{array}$ & $\begin{array}{c}9.50 \\
(3.16)\end{array}$ \\
\hline $\mathbf{T}_{3}$ & $\begin{array}{c}7.12 \\
(2.76)\end{array}$ & $\begin{array}{c}8.00 \\
(2.91)\end{array}$ & $\begin{array}{c}8.87 \\
(3.06)\end{array}$ & $\begin{array}{c}8.00 \\
(2.91)\end{array}$ & $\begin{array}{c}8.75 \\
(3.04)\end{array}$ & $\begin{array}{c}9.12 \\
(3.10)\end{array}$ & $\begin{array}{c}9.50 \\
(3.16)\end{array}$ & $\begin{array}{c}9.12 \\
(3.10)\end{array}$ & $\begin{array}{c}7.62 \\
(2.84)\end{array}$ & $\begin{array}{c}9.00 \\
(3.08)\end{array}$ & $\begin{array}{l}10.35 \\
(3.29)\end{array}$ & $\begin{array}{c}8.99 \\
(3.08)\end{array}$ & $\begin{array}{c}8.75 \\
(3.04)\end{array}$ & $\begin{array}{c}9.50 \\
(3.16)\end{array}$ & $\begin{array}{l}10.62 \\
(3.33)\end{array}$ & $\begin{array}{c}9.62 \\
(3.18)\end{array}$ \\
\hline $\mathbf{T}_{4}$ & \multicolumn{3}{|c|}{$\begin{array}{c}0.00 \\
(0.70)\end{array}$} & $\begin{array}{c}0.00 \\
(0.70)\end{array}$ & \multicolumn{3}{|c|}{$\begin{array}{c}0.00 \\
(0.70)\end{array}$} & $\begin{array}{c}0.00 \\
(0.70)\end{array}$ & \multicolumn{3}{|c|}{$\begin{array}{c}0.00 \\
(0.70)\end{array}$} & $\begin{array}{c}0.00 \\
(0.70)\end{array}$ & \multicolumn{3}{|c|}{$\begin{array}{c}0.00 \\
(0.70)\end{array}$} & $\begin{array}{c}0.00 \\
(0.70)\end{array}$ \\
\hline Mean & $\begin{array}{c}5.44 \\
(2.43)\end{array}$ & $\begin{array}{c}5.81 \\
(2.51)\end{array}$ & $\begin{array}{c}6.37 \\
(2.62)\end{array}$ & $\begin{array}{c}5.87 \\
(2.52)\end{array}$ & $\begin{array}{c}6.00 \\
(2.54)\end{array}$ & $\begin{array}{c}6.53 \\
(2.65)\end{array}$ & $\begin{array}{c}7.03 \\
(2.74)\end{array}$ & $\begin{array}{c}6.52 \\
(2.64)\end{array}$ & $\begin{array}{c}5.81 \\
(2.51)\end{array}$ & $\begin{array}{c}6.45 \\
(2.63)\end{array}$ & $\begin{array}{c}7.40 \\
(2.81)\end{array}$ & $\begin{array}{c}6.55 \\
(2.65)\end{array}$ & $\begin{array}{c}6.31 \\
(2.61)\end{array}$ & $\begin{array}{c}6.97 \\
(2.73)\end{array}$ & $\begin{array}{c}7.77 \\
(2.87)\end{array}$ & $\begin{array}{c}7.02 \\
(2.74)\end{array}$ \\
\hline \multirow[t]{2}{*}{ F test } & \multicolumn{2}{|c|}{ Dilutions } & \multicolumn{3}{|c|}{ Forms } & \multicolumn{3}{|c|}{ Concentrations } & \multicolumn{2}{|c|}{ Dilutions } & \multicolumn{3}{|c|}{ Forms } & \multicolumn{3}{|c|}{ Concentrations } \\
\hline & * & * & & $*$ & & & $*$ & & * & * & & $*$ & & & $*$ & \\
\hline S. Em \pm & $\begin{array}{r}0.1 \\
(0.0\end{array}$ & $\begin{array}{l}31 \\
22)\end{array}$ & & $\begin{array}{c}0.278 \\
(0.046)\end{array}$ & & & $\begin{array}{c}0.131 \\
(0.022)\end{array}$ & & $\begin{array}{r}0.0 \\
(0.0\end{array}$ & $\begin{array}{l}91 \\
15)\end{array}$ & & $\begin{array}{c}0.193 \\
(0.031)\end{array}$ & & & $\begin{array}{c}0.091 \\
(0.015)\end{array}$ & \\
\hline CD 5\% & $\begin{array}{r}0.3 \\
(0.0\end{array}$ & $\begin{array}{l}62 \\
60)\end{array}$ & & $\begin{array}{c}0.768 \\
(0.126)\end{array}$ & & & $\begin{array}{c}0.362 \\
(0.060)\end{array}$ & & $\begin{array}{r}0.2 \\
(0.0\end{array}$ & $\begin{array}{l}251 \\
41)\end{array}$ & & $\begin{array}{c}0.533 \\
(0.086)\end{array}$ & & & $\begin{array}{c}0.251 \\
(0.041)\end{array}$ & \\
\hline
\end{tabular}

$\mathrm{T}_{1}$ : Aqueous form + Spraying, $\mathrm{T}_{2}$ : Acetone form + Spraying, $\mathrm{T}_{3}:$ Alcohol form + Spraying, $\mathrm{T}_{4}:$ Water; $\quad *$ - Significant 
Table.2 Zone of inhibition ( $\mathrm{mm}$ ) observed in different concentrations (2\%, $4 \%$ and $6 \%)$ of Adhatoda vasica against Staphylococcus sp.

\begin{tabular}{|c|c|c|c|c|c|c|c|c|c|c|c|c|c|c|c|c|}
\hline \multirow{4}{*}{\begin{tabular}{|l|} 
Dilution \\
$\begin{array}{c}\text { Conc. of } \\
\text { botanical }\end{array}$ \\
$\begin{array}{l}\text { Forms of } \\
\text { botanical }\end{array}$ \\
\end{tabular}} & \multicolumn{8}{|c|}{24 hours } & \multicolumn{8}{|c|}{48 hours } \\
\hline & \multicolumn{4}{|c|}{$10^{-5}$} & \multicolumn{4}{|c|}{$10^{-7}$} & \multicolumn{4}{|c|}{$10^{-5}$} & \multicolumn{4}{|c|}{$10^{-7}$} \\
\hline & \multirow[t]{2}{*}{$2 \%$} & \multirow[t]{2}{*}{$4 \%$} & \multirow[t]{2}{*}{$6 \%$} & \multirow[t]{2}{*}{ Mean } & \multirow[t]{2}{*}{$2 \%$} & \multirow[t]{2}{*}{$4 \%$} & \multirow[t]{2}{*}{$6 \%$} & \multirow[t]{2}{*}{ Mean } & \multirow[t]{2}{*}{$2 \%$} & \multirow[t]{2}{*}{$4 \%$} & \multirow[t]{2}{*}{$6 \%$} & \multirow[t]{2}{*}{ Mean } & \multirow[t]{2}{*}{$2 \%$} & \multirow[t]{2}{*}{$4 \%$} & \multirow[t]{2}{*}{$6 \%$} & \multirow[t]{2}{*}{ Mean } \\
\hline & & & & & & & & & & & & & & & & \\
\hline $\mathrm{T}_{1}$ & $\begin{array}{c}6.62 \\
(2.66)\end{array}$ & $\begin{array}{c}6.88 \\
(2.71)\end{array}$ & $\begin{array}{c}7.38 \\
(2.80)\end{array}$ & $\begin{array}{c}6.96 \\
(2.73)\end{array}$ & $\begin{array}{c}6.88 \\
(2.71)\end{array}$ & $\begin{array}{c}7.25 \\
(2.78)\end{array}$ & $\begin{array}{c}7.75 \\
(2.87)\end{array}$ & $\begin{array}{c}7.29 \\
(2.79)\end{array}$ & $\begin{array}{c}6.88 \\
(2.71)\end{array}$ & $\begin{array}{c}7.38 \\
(2.80)\end{array}$ & $\begin{array}{c}7.8 \\
(2.88)\end{array}$ & $\begin{array}{c}7.35 \\
(2.80)\end{array}$ & $\begin{array}{c}7.25 \\
(2.78)\end{array}$ & $\begin{array}{c}7.63 \\
(2.85)\end{array}$ & $\begin{array}{c}8.5 \\
(3.00)\end{array}$ & $\begin{array}{c}7.79 \\
(2.87)\end{array}$ \\
\hline $\mathbf{T}_{2}$ & $\begin{array}{c}6.50 \\
(2.64)\end{array}$ & $\begin{array}{c}7.13 \\
(2.76)\end{array}$ & $\begin{array}{c}7.50 \\
(2.82)\end{array}$ & $\begin{array}{c}7.04 \\
(2.74)\end{array}$ & $\begin{array}{c}7.13 \\
(2.76)\end{array}$ & $\begin{array}{c}7.50 \\
(2.82)\end{array}$ & $\begin{array}{c}8.13 \\
(2.93)\end{array}$ & $\begin{array}{c}7.59 \\
(2.84)\end{array}$ & $\begin{array}{c}7.13 \\
(2.76)\end{array}$ & $\begin{array}{c}7.50 \\
(2.82)\end{array}$ & $\begin{array}{l}8.25 \\
(2.95)\end{array}$ & $\begin{array}{c}7.63 \\
(2.85)\end{array}$ & $\begin{array}{c}7.53 \\
(2.83)\end{array}$ & $\begin{array}{c}8.50 \\
(3.00)\end{array}$ & $\begin{array}{c}8.75 \\
(3.04)\end{array}$ & $\begin{array}{c}8.26 \\
(2.96)\end{array}$ \\
\hline $\mathbf{T}_{\mathbf{3}}$ & $\begin{array}{c}6.75 \\
(2.69)\end{array}$ & $\begin{array}{c}7.50 \\
(2.82)\end{array}$ & $\begin{array}{c}8.00 \\
(2.91)\end{array}$ & $\begin{array}{c}7.42 \\
(2.81)\end{array}$ & $\begin{array}{c}7.38 \\
(2.80)\end{array}$ & $\begin{array}{c}7.88 \\
(2.89)\end{array}$ & $\begin{array}{c}8.38 \\
(2.97)\end{array}$ & $\begin{array}{c}7.88 \\
(2.89)\end{array}$ & $\begin{array}{c}7.13 \\
(2.76)\end{array}$ & $\begin{array}{c}7.63 \\
(2.85)\end{array}$ & $\begin{array}{c}8.75 \\
(3.04)\end{array}$ & $\begin{array}{c}7.84 \\
(2.88)\end{array}$ & $\begin{array}{c}8.13 \\
(2.93)\end{array}$ & $\begin{array}{c}8.13 \\
(2.93)\end{array}$ & $\begin{array}{c}9.62 \\
(3.18)\end{array}$ & $\begin{array}{c}8.63 \\
(3.02)\end{array}$ \\
\hline $\mathbf{T}_{4}$ & \multicolumn{3}{|c|}{$\begin{array}{c}0.00 \\
(0.70)\end{array}$} & $\begin{array}{c}0.00 \\
(0.70)\end{array}$ & \multicolumn{3}{|c|}{$\begin{array}{c}0.00 \\
(0.70)\end{array}$} & $\begin{array}{c}0.00 \\
(0.70)\end{array}$ & \multicolumn{3}{|c|}{$\begin{array}{c}0.00 \\
(0.70)\end{array}$} & $\begin{array}{c}0.00 \\
(0.70)\end{array}$ & \multicolumn{3}{|c|}{$\begin{array}{c}0.00 \\
(0.70)\end{array}$} & $\begin{array}{c}0.00 \\
(0.70)\end{array}$ \\
\hline Mean & $\begin{array}{c}4.97 \\
(2.33)\end{array}$ & $\begin{array}{c}5.38 \\
(2.42)\end{array}$ & $\begin{array}{c}5.72 \\
(2.49)\end{array}$ & $\begin{array}{c}5.36 \\
(2.41)\end{array}$ & $\begin{array}{c}5.35 \\
(2.41)\end{array}$ & $\begin{array}{c}5.66 \\
(2.48)\end{array}$ & $\begin{array}{c}6.07 \\
(2.56)\end{array}$ & $\begin{array}{c}5.69 \\
(2.48)\end{array}$ & $\begin{array}{l}5.29 \\
(2.40)\end{array}$ & $\begin{array}{c}5.63 \\
(2.47)\end{array}$ & $\begin{array}{c}6.20 \\
(2.58)\end{array}$ & $\begin{array}{c}5.70 \\
(2.49)\end{array}$ & $\begin{array}{c}5.73 \\
(2.49)\end{array}$ & $\begin{array}{c}6.06 \\
(2.56)\end{array}$ & $\begin{array}{c}6.72 \\
(2.68)\end{array}$ & $\begin{array}{c}6.17 \\
(2.58)\end{array}$ \\
\hline \multirow[t]{2}{*}{ F test } & \multicolumn{2}{|c|}{ Dilutions } & \multicolumn{3}{|c|}{ Forms } & \multicolumn{3}{|c|}{ Concentrations } & \multicolumn{2}{|c|}{ Dilutions } & \multicolumn{3}{|c|}{ Forms } & \multicolumn{3}{|c|}{ Concentrations } \\
\hline & & $*$ & & $*$ & & & $*$ & & & $*$ & & $*$ & & & $*$ & \\
\hline S. Em \pm & $\begin{array}{r}0.0 \\
(0.0\end{array}$ & 62 & & $\begin{array}{c}0.132 \\
(0.024)\end{array}$ & & & $\begin{array}{c}0.062 \\
(0.011)\end{array}$ & & $\begin{array}{r}0.0 \\
(0.0\end{array}$ & $\begin{array}{l}043 \\
007)\end{array}$ & & $\begin{array}{c}0.092 \\
(0.016)\end{array}$ & & & $\begin{array}{c}0.043 \\
(0.007)\end{array}$ & \\
\hline CD $5 \%$ & $\begin{array}{r}0.1 \\
(0.0\end{array}$ & 172 & & $\begin{array}{c}0.365 \\
(0.066)\end{array}$ & & & $\begin{array}{c}0.172 \\
(0.031)\end{array}$ & & & $\begin{array}{l}120 \\
020)\end{array}$ & & $\begin{array}{c}0.254 \\
(0.043)\end{array}$ & & & $\begin{array}{l}0.120 \\
(0.020)\end{array}$ & \\
\hline
\end{tabular}

$\mathrm{T}_{1}$ : Aqueous form + Spraying, $\mathrm{T}_{2}$ : Acetone form + Spraying, $\mathrm{T}_{3}$ : Alcohol form + Spraying, $\mathrm{T}_{4}:$ Water; ${ }^{*}-$ Significant 
Table.3 Zone of inhibition (mm) observed in different concentrations (2\%, $4 \%$ and 6\%) of Adhatoda vasica against Streptococcus sp.

\begin{tabular}{|c|c|c|c|c|c|c|c|c|c|c|c|c|c|c|c|c|}
\hline \multirow{4}{*}{\begin{tabular}{|l|} 
Dilution \\
$\begin{array}{l}\text { Conc. of } \\
\text { botanical }\end{array}$ \\
$\begin{array}{l}\text { Forms of } \\
\text { botanical }\end{array}$ \\
\end{tabular}} & \multicolumn{8}{|c|}{24 hours } & \multicolumn{8}{|c|}{48 hours } \\
\hline & \multicolumn{4}{|c|}{$10^{-5}$} & \multicolumn{4}{|c|}{$10^{-7}$} & \multicolumn{4}{|c|}{$10^{-5}$} & \multicolumn{4}{|c|}{$10^{-7}$} \\
\hline & \multirow[t]{2}{*}{$2 \%$} & \multirow[t]{2}{*}{$4 \%$} & \multirow[t]{2}{*}{$6 \%$} & \multirow[t]{2}{*}{ Mean } & \multirow[t]{2}{*}{$2 \%$} & \multirow[t]{2}{*}{$4 \%$} & \multirow[t]{2}{*}{$6 \%$} & \multirow[t]{2}{*}{ Mean } & \multirow[t]{2}{*}{$2 \%$} & \multirow[t]{2}{*}{$4 \%$} & \multirow[t]{2}{*}{$6 \%$} & \multirow[t]{2}{*}{ Mean } & \multirow[t]{2}{*}{$2 \%$} & \multirow[t]{2}{*}{$4 \%$} & \multirow[t]{2}{*}{$6 \%$} & \multirow[t]{2}{*}{ Mean } \\
\hline & & & & & & & & & & & & & & & & \\
\hline $\mathbf{T}_{1}$ & $\begin{array}{c}5.75 \\
(2.50)\end{array}$ & $\begin{array}{c}6.62 \\
(2.66)\end{array}$ & $\begin{array}{c}7.00 \\
(2.73)\end{array}$ & $\begin{array}{c}6.46 \\
(2.63)\end{array}$ & $\begin{array}{c}6.50 \\
(2.64)\end{array}$ & $\begin{array}{c}7.00 \\
(2.73)\end{array}$ & $\begin{array}{c}7.25 \\
(2.78)\end{array}$ & $\begin{array}{c}6.92 \\
(2.73)\end{array}$ & $\begin{array}{c}7.63 \\
(2.85)\end{array}$ & $\begin{array}{c}8.00 \\
(2.91)\end{array}$ & $\begin{array}{c}8.63 \\
(3.02)\end{array}$ & $\begin{array}{c}8.09 \\
(2.93)\end{array}$ & $\begin{array}{c}7.75 \\
(2.87)\end{array}$ & $\begin{array}{c}8.38 \\
(2.98)\end{array}$ & $\begin{array}{c}9.25 \\
(3.12)\end{array}$ & $\begin{array}{c}8.46 \\
(2.99)\end{array}$ \\
\hline $\mathbf{T}_{2}$ & $\begin{array}{l}7.50 \\
(2.82)\end{array}$ & $\begin{array}{c}7.62 \\
(2.84)\end{array}$ & $\begin{array}{c}8.25 \\
(2.95)\end{array}$ & $\begin{array}{c}7.79 \\
(2.87)\end{array}$ & $\begin{array}{c}7.50 \\
(2.82)\end{array}$ & $\begin{array}{l}8.12 \\
(2.93)\end{array}$ & $\begin{array}{c}8.75 \\
(3.04)\end{array}$ & $\begin{array}{c}8.12 \\
(2.93)\end{array}$ & $\begin{array}{c}7.75 \\
(2.87)\end{array}$ & $\begin{array}{c}8.50 \\
(3.00)\end{array}$ & $\begin{array}{c}9.25 \\
(3.12)\end{array}$ & $\begin{array}{c}8.50 \\
(3.00)\end{array}$ & $\begin{array}{c}8.38 \\
(2.97)\end{array}$ & $\begin{array}{c}8.50 \\
(3.00)\end{array}$ & $\begin{array}{c}9.75 \\
(3.20)\end{array}$ & $\begin{array}{c}8.88 \\
(3.06)\end{array}$ \\
\hline $\mathbf{T}_{3}$ & $\begin{array}{c}7.62 \\
(2.84)\end{array}$ & $\begin{array}{c}8.37 \\
(2.97)\end{array}$ & $\begin{array}{c}8.75 \\
(3.04)\end{array}$ & $\begin{array}{c}8.25 \\
(2.95)\end{array}$ & $\begin{array}{c}8.00 \\
(2.91)\end{array}$ & $\begin{array}{c}8.62 \\
(3.01)\end{array}$ & $\begin{array}{c}10.00 \\
(3.24)\end{array}$ & $\begin{array}{c}8.87 \\
(3.06)\end{array}$ & $\begin{array}{c}8.00 \\
(2.91)\end{array}$ & $\begin{array}{c}8.63 \\
(3.02)\end{array}$ & $\begin{array}{l}9.88 \\
(3.22)\end{array}$ & $\begin{array}{c}8.84 \\
(3.05)\end{array}$ & $\begin{array}{c}8.50 \\
(3.00)\end{array}$ & $\begin{array}{c}8.88 \\
(3.06)\end{array}$ & $\begin{array}{l}10.25 \\
(3.27)\end{array}$ & $\begin{array}{c}9.21 \\
(3.11)\end{array}$ \\
\hline $\mathbf{T}_{4}$ & \multicolumn{3}{|c|}{$\begin{array}{c}0.00 \\
(0.70)\end{array}$} & $\begin{array}{c}0.00 \\
(0.70)\end{array}$ & \multicolumn{3}{|c|}{$\begin{array}{c}0.00 \\
(0.70)\end{array}$} & $\begin{array}{c}0.00 \\
(0.70)\end{array}$ & \multicolumn{3}{|c|}{$\begin{array}{c}0.00 \\
(0.70)\end{array}$} & $\begin{array}{c}0.00 \\
(0.70)\end{array}$ & \multicolumn{3}{|c|}{$\begin{array}{c}0.00 \\
(0.70)\end{array}$} & $\begin{array}{c}0.00 \\
(0.70)\end{array}$ \\
\hline Mean & $\begin{array}{c}5.22 \\
(2.39)\end{array}$ & $\begin{array}{c}5.65 \\
(2.48)\end{array}$ & $\begin{array}{c}6.00 \\
(2.54)\end{array}$ & $\begin{array}{c}5.62 \\
(2.47)\end{array}$ & $\begin{array}{c}5.50 \\
(2.44)\end{array}$ & $\begin{array}{c}5.94 \\
(2.53)\end{array}$ & $\begin{array}{c}6.50 \\
(2.64)\end{array}$ & $\begin{array}{c}5.98 \\
(2.54)\end{array}$ & $\begin{array}{c}5.85 \\
(2.51)\end{array}$ & $\begin{array}{c}6.28 \\
(2.60)\end{array}$ & $\begin{array}{c}6.94 \\
(2.73)\end{array}$ & $\begin{array}{c}6.36 \\
(2.61)\end{array}$ & $\begin{array}{c}6.16 \\
(2.58)\end{array}$ & $\begin{array}{c}6.44 \\
(2.63)\end{array}$ & $\begin{array}{c}7.31 \\
(2.79)\end{array}$ & $\begin{array}{c}6.64 \\
(2.67)\end{array}$ \\
\hline \multirow[t]{2}{*}{ F test } & \multicolumn{2}{|c|}{ Dilutions } & \multicolumn{3}{|c|}{ Concentrations } & \multicolumn{3}{|c|}{ Forms } & \multicolumn{2}{|c|}{ Dilutions } & \multicolumn{3}{|c|}{ Concentrations } & \multicolumn{3}{|c|}{ Forms } \\
\hline & $x$ & k & & $*$ & & & $*$ & & * & 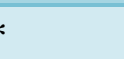 & & $*$ & & & $*$ & \\
\hline S. Em \pm & $\begin{array}{r}0.0 \\
(0.0\end{array}$ & $\begin{array}{l}72 \\
13)\end{array}$ & & $\begin{array}{c}0.152 \\
(0.027)\end{array}$ & & & $\begin{array}{c}0.072 \\
(0.013)\end{array}$ & & $\begin{array}{r}0.0 \\
(0.0\end{array}$ & $\begin{array}{l}66 \\
11)\end{array}$ & & $\begin{array}{c}0.141 \\
(0.023)\end{array}$ & & & $\begin{array}{c}0.066 \\
(0.011)\end{array}$ & \\
\hline CD $5 \%$ & $\begin{array}{r}0.1 \\
(0.0\end{array}$ & $\begin{array}{l}98 \\
35)\end{array}$ & & $\begin{array}{c}0.420 \\
(0.073)\end{array}$ & & & $\begin{array}{c}0.198 \\
(0.035)\end{array}$ & & $\begin{array}{r}0.1 \\
(0.0\end{array}$ & $\begin{array}{l}84 \\
30)\end{array}$ & & $\begin{array}{c}0.389 \\
(0.064)\end{array}$ & & & $\begin{array}{c}0.184 \\
(0.030)\end{array}$ & \\
\hline
\end{tabular}

$\mathrm{T}_{1}$ : Aqueous form + Spraying, $\mathrm{T}_{2}:$ Acetone form + Spraying, $\mathrm{T}_{3}:$ Alcohol form + Spraying, $\mathrm{T}_{4}:$ Water; ${ }^{*}-$ Significant 


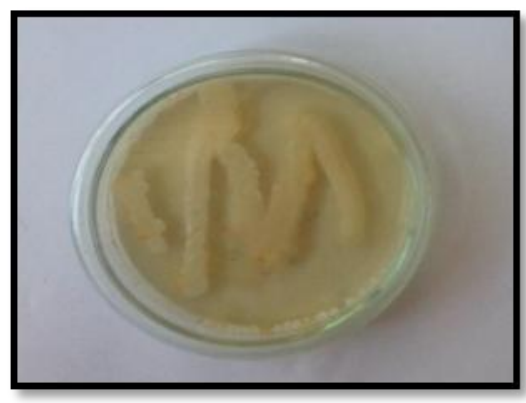

Bacillus sp.

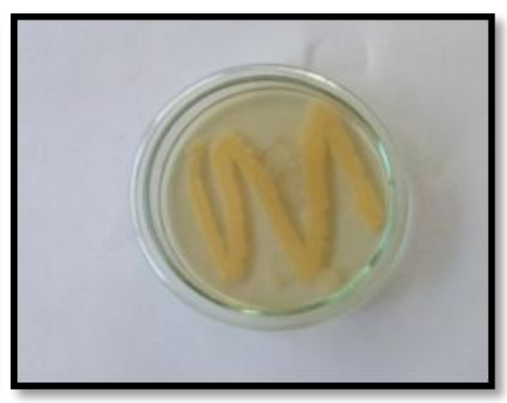

Staphylococcus sp.

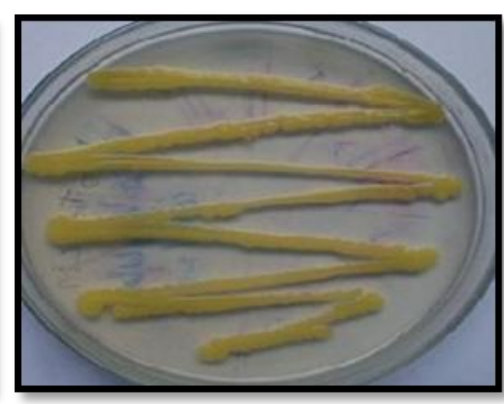

Streptococcus sp.

Fig.1 Bacterial species isolated from silkworm haemolymph
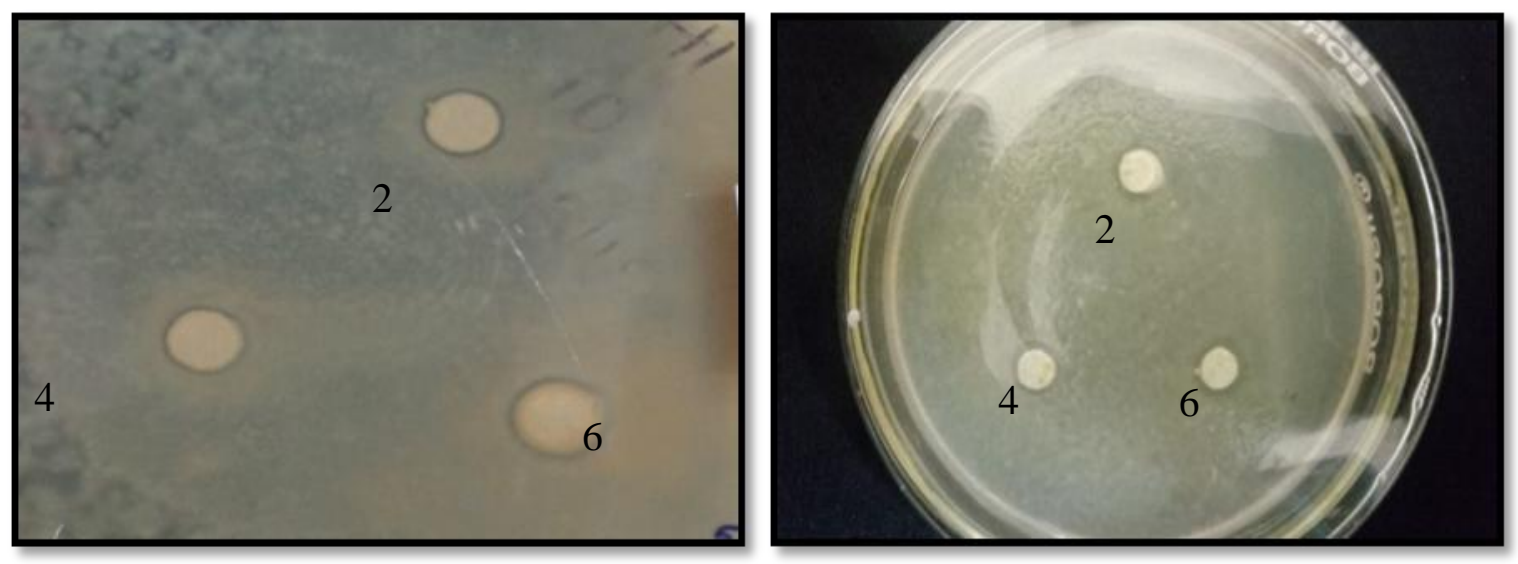

Aqueous form+Spraying
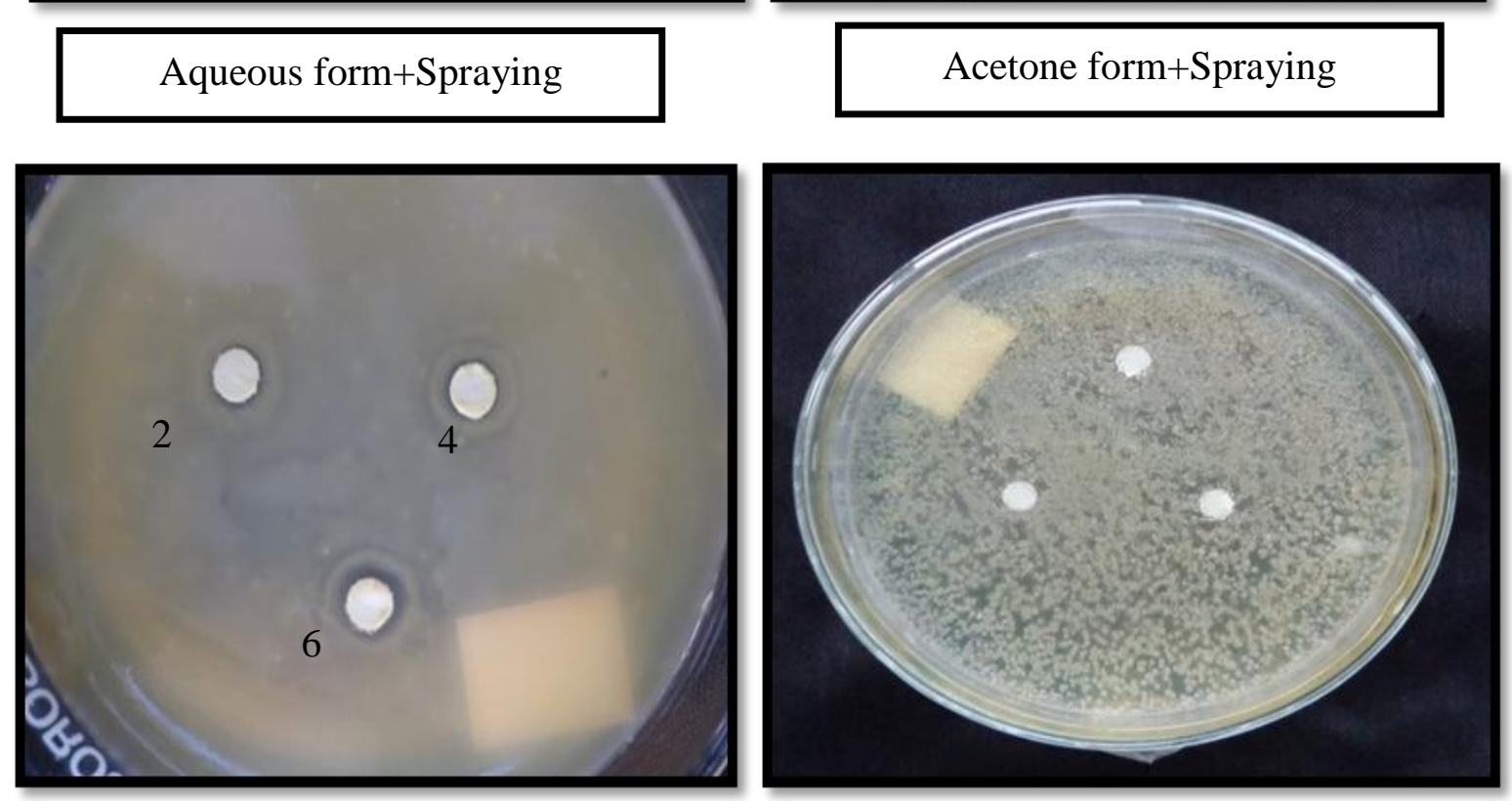

Alcoholic form+Spraying

Water

Fig.2 Inhibition zone of Bacillus sp. exhibited by Adhatoda vasica 
The trend was observed similar with other liquid forms $\mathrm{T}_{2}$ (Acetone form + spraying) (7.04 and 7.59., 7.63 and $8.26 \mathrm{~mm}$ ) and $\mathrm{T}_{1}$ (aqueous form + spraying) (6.96 and 7.29., 7.35 and $7.79 \mathrm{~mm}$ ) and found significant in $10^{-5}$ and $10^{-7}$ dilution at 24 and 48 hours of incubation respectively compared to control batches (Table 2).

These results were found on par with the findings of (Hossain et al., 2013), according to them, under in-vitro study the use of methanol, petroleum spirit, ethyl acetate and dichloromethane extracts of Tinospora cordifolia were found effective against Bacillus subtilis and Escherichia coli. The maximum zone of inhibition (11.33 and 8.33 $\mathrm{mm}$ ) was recorded for $E$. coli in methanol and ethyl acetate, whereas zone of inhibition of 10.67 and $8.00 \mathrm{~mm}$ was observed for $B$. subtilis with petroleum spirit and dichloromethane extracts of Tinospora cordifolia. In addition, minimum zone (6.67 $\mathrm{mm}$ ) was reported fordichloro-methane and ethyl acetate extracts used against $E$. coli and B. subtilis.

Zone of inhibition ( $\mathrm{mm}$ ) observed in different concentrations $(2 \%, 4 \%$ and 6 $\%$ of Adhatoda vasica against Streptococcus sp.

The application of botanical extracts of A.vasica at different concentrations against Streptococcus sp. revealed significant differences pertaining to forms and methods of botanical application, different concentrations of botanicals and different bacterial dilutions at 24 hours of incubation. Further, significant differences were noticed among the treatments viz., $\mathrm{T}_{1}(6.46$ and 6.92 $\mathrm{mm}), \mathrm{T}_{2}(7.79$ and $8.12 \mathrm{~mm})$ and $\mathrm{T}_{3}(8.25$ and $8.87 \mathrm{~mm})$ over water control $\mathrm{T}_{4}(0.00 \mathrm{~mm})$ after 24 hours of incubation. In addition, the significant difference was also noticed in between the per cent botanical concentration viz., 2 (5.22 and $5.50 \mathrm{~mm}), 4$ (5.65 and 5.50 $\mathrm{mm})$ and $6(6.00$ and $6.50 \mathrm{~mm})$ at both the bacterial dilutions of $10^{-5}$ and $10^{-7}$ respectively (Table 3).

The zone of inhibition after 48 hours of incubation recorded significant difference between liquid forms $\left(\mathrm{T}_{1}-8.09, \mathrm{~T}_{2}-8.50\right.$ and $\left.\mathrm{T}_{3}-8.84 \mathrm{~mm}\right)$ and $\left(\mathrm{T}_{1}-8.46, \mathrm{~T}_{2}-8.88\right.$ and $\mathrm{T}_{3^{-}}$ $9.21 \mathrm{~mm}$ ) and the results were on par with each other against $10^{-5}$ dilution whereas at $10^{-}$ ${ }^{7}$ dilutions all the forms and methods resulted in significant differences in inhibiting growth of bacteria (Table 3 ).

It is further confirmed by (Samatha et al., 2013) that, at 1:1 concentration, the aqueous extract of Oroxylum indicum showed greater inhibition of $11.7 \mathrm{~mm}$ (Bacillus subtilis), 8.7 $\mathrm{mm} \quad$ (Staphylococcus aureus), 6.6 $\mathrm{mm}$ (Staphylococcus albus) whereas at 1:2 concentration, maximum zone of inhibition $(5.2 \mathrm{~mm})$ was found in Bacillus cereus when compared to the control (6.3, $6.2,4$ and $3 \mathrm{~mm}$ ).

The in-vitro effect of Adhatoda vasica at different concentrations, methods and forms of application were revealed, significant difference and found effective against all the three bacterial species. To assess the effectiveness of these botanical formulations against Bacillus sp., Staphylococcus sp. and Streptococcus sp., the zone of inhibition was assessed. Among three bacterial species, the maximum zone of inhibition was observed in alcoholic extract on 24 and 48 hours of incubation against Bacillus sp. at $10^{-5}$ and $10^{-7}$ spore dilution.

The trend was same in other bacterial species viz., Staphylococcus and Streptococcus. However the increased zone of inhibition was observed on 24 and 48 hours of incubation and on 72 hours onwards, the inhibition was found decreased due to the loss of 
antibacterial components present in the botanical extracts might have decrease the property of botanical that leads to increase in all the three types of bacterial growth.

\section{References}

Chatterjee, A. and Pakrashi, S. C. 1991.The treatise on Indian medicinal plants. I, XII-XX publications and information directorate, New Delhi: Pp:14-15.

Chopra, R. N. 1982.Indigenous drugs of India, $2^{\text {nd }}$ ed. Academic publishers, Calcutta.Pp: 72-74.

Karthikairaj, K., Prasannakumar, K. andIsaiarasu, L. 2013. Use of plant extracts for the control of flacherie disease in silkworm, Bombyxmori L. (Lepidoptera: Bombicidae). Int. J. Microbiol. Res., 4(2): 158-161

Krishna Prasad, K. S., Siddaramaiah, A. L. andLingaraju, S. 1979. Possible control of muscardine disease of silkworms by using a few plant extracts. Curr. Res.,8(5): 79-80.

Nataraju, B., Sivaprasad, V. andDatta, R. K. 1999. Studies on the cause of
Thatteroga in silkworm, BombyxmoriL. Indian J. Seric.,38. Pp: 149-151.

Ray, P. G. and Majumdar, S. K.1974.Antimicrobial activity of some Indian plants. Economic Botany, 30: Pp: 317-320.

SamathaTalari, Sampath, A., Sujatha, K. and RamaSwamyNanna.2013.Antibacterial Activity of Stem Bark Extracts of Oroxylumindicuman Endangered Ethnomedicinal Forest Tree. J. Pharmacy and Biol. Sci.,7(1): 24-28.

ShamimHossain, TouhidaKhatun and Mehedi H. 2013.In-vitro bacterial effect of Tinospoacordifolia extracts against some selective bacterial pathogens. Int. J. Biosci., 3(7):156-161.

Sironmani, A. T., Meena, P. and Vanitha Rani, R. 1994. Isolation and characterization of pathogenic bacterial species in the silkworm, Bombyxmori L. Sericologia,34: 97-102.

Sugun, R. 2000.Status and management of Thatte disease of silkworm. Advances in Tropical Sericulture. Proc. Natl. Sem. Tropic. Seric.,UAS, GKVK, Bengaluru. Pp. 84-86.

\section{How to cite this article:}

Anitharani. K. V., R. N. Bhaskar and Anusha. H. G. 2020. In-Vitro Efficacy of Forms and Methods of Adhatoda vasica against Flacherie causing Bacteria of Silkworm, Bombyx mori L. Int.J.Curr.Microbiol.App.Sci. 9(07): 346-354. doi: https://doi.org/10.20546/ijcmas.2020.907.037 